\title{
Control of interfacial instabilities using
}

\section{flow geometry}

\author{
Talal T. Al-Housseiny ${ }^{1}$, Peichun A. Tsai ${ }^{2}$ and Howard A. Stone ${ }^{2 \star}$
}

The displacement of one fluid by another is one of the most common processes involving interfacial instabilities. It is universally accepted that, in a uniform medium, flow displacement is unstable when a low-viscosity fluid invades a fluid of higher viscosity: the classical viscous fingering instability ${ }^{1-4}$. Consequently, once fluid properties are specified, opportunities for control become very limited. However, real systems where displacement instabilities occur, such as porous structures $^{5,6}$, lung airways $s^{7,8}$ and printing devices ${ }^{9-11}$, are rarely uniform. We find that the simplest heterogeneity-a gradient in the flow passage ${ }^{12-15}$ - can lead to fundamentally different displacement behaviours. We use this finding to either inhibit or trigger an instability and, hence, to devise a strategy to manipulate instabilities in fluid-fluid systems. The control setting we identify has a wide spectrum of applications ranging from small-scale technologies such as microfluidics to largescale operations such as enhanced oil recovery.

Interfacial instabilities impact, to give a few examples, the dendritic shape of snow flakes ${ }^{16}$, early failure in zinc alkaline batteries ${ }^{17}$, breath sounds caused by surfactant deficiency in lungs ${ }^{7}$ and the protection of the stomach from its own gastric acids ${ }^{18}$. Morphological patterns and periodic structures are often the form in which interfacial instabilities are manifested. For instance, patterns are detected on the surface of metal casts due to shrinkage on solidification of the metal ${ }^{19,20}$, and the wavy interface of lubrication oil confined in journal bearings and between printing rollers is a signature of the so-called printer's instability ${ }^{9-11}$. In most cases, interfacial instabilities hinder the operation of processes and limit their efficiency. Moreover, instabilities at the interface of two distinct fluids remain a major challenge for enhanced oil recovery processes such as water flooding ${ }^{21}$. On the other hand, these instabilities can be beneficial to chromatographic separation and can improve mixing in non-turbulent systems and small-scale devices $^{22}$. The fact that depending on the application either a stable or an unstable interface is desirable makes the ability to control interfacial instabilities ${ }^{23,24}$ essential in design and technology.

In the early twentieth century, petroleum and mining engineers noticed that water does not displace oil uniformly ${ }^{25}$. Rather, water penetrates through oil, a phenomenon now known as fingering owing to the finger-like interfacial patterns. The stability of the interface between the invading and the displaced fluids was first analysed in the 1950s (refs 1,5,26). Most notably, Saffman and Taylor found ${ }^{1}$ that when a fluid of low viscosity invades a fluid of higher viscosity in the narrow space confined between two parallel plates (a Hele-Shaw cell), the interface is always unstable. The instability, which occurs at any flow speed, takes the form of propagating narrow fingers of the low-viscosity fluid, leaving behind the more viscous fluid. Conversely, in a uniform Hele-Shaw cell, a high-viscosity fluid displacing a less viscous fluid leads to a flat stable interface that propagates uniformly. Fingering instabilities in porous media flows have since been studied extensively ${ }^{1-6}$ owing to their importance in enhanced oil recovery ${ }^{21,27,28}$ and the increasing interest in carbon sequestration ${ }^{29}$.

The classical two-dimensional Hele-Shaw configuration constitutes a convenient framework ${ }^{2}$ to study interfacial instabilities in narrow passages. Nevertheless, whether these passages consist of small clearances in journal bearings, lung airways or channels in microfluidic chips, they are rarely homogeneous or uniform. Is it possible to take advantage of a given channel shape to control instabilities or, even better, to design a system such that a less viscous fluid can stably displace a more viscous fluid? Motivated by the importance of controlling interfacial instabilities, and to mimic a degree of heterogeneity of a real system, we investigate a variant of the classical viscous fingering problem by introducing a small depth gradient in the Hele-Shaw cell (see Fig. 1a). In our experiments, the two, nearly horizontal, plates are at a small angle with respect to each other ${ }^{12,30}$; the depth of the cell varies linearly along the direction of fluid displacement, so the flow is either converging or diverging.

As expected, when air displaces mineral oil (viscosity $\mu_{1}=25 \mathrm{cP}$, surface tension $\gamma=29 \mathrm{mN} \mathrm{m}^{-1}$ ) in a horizontal Hele-Shaw cell with a constant gap, the interface is unstable and takes the shape of a finger that occupies half the width of the cell; Fig. 1b shows our experiment of this well-known fingering instability. However, we have discovered that if the same kind of experiment, air propagating into oil, is conducted in a converging cell (negative gradient, $\alpha<0$ ), that is, the cell becomes shallower in the direction of flow, the interface can become stable. For example, a snapshot of our experiment of a stable flat interface propagating in a converging cell is shown in Fig. 1c.

A series of systematic experiments was performed (Fig. 2) to examine the effect of the gradient on the air-oil interface. The air-flow rate, characterized by an average inlet velocity $U$ (see Fig. 1a), was controlled by applying a constant pressure drop across the cell (see Supplementary Information for details). In this way, we varied the characteristic capillary number $\mathrm{Ca}=12 \mu_{1} U / \gamma$, which is the ratio of viscous forces to surface tension forces. The interface of this system is classically unstable $\mathrm{e}^{1,2,5}$ because air is much less viscous than mineral oil. Nevertheless, in the presence of a negative depth gradient, fingering is inhibited and the interface becomes stable (Fig. 2b) below a critical capillary number $\mathrm{Ca}_{c}$; see Fig. 2d. Above the $\mathrm{Ca}_{\mathrm{c}}$ (Fig. 2c), and in contrast with classical experiments, the gradient here resulted in wider air fingers ${ }^{12}$ that occupy more than half of the cell width (see Supplementary Information).

To understand the transition in interfacial behaviour observed in the experiments and to explain the critical capillary number, we analyse the onset of the instability in the presence of the gradient. The Hele-Shaw cell is characterized by its depth $h(x)=h_{0}+\alpha x$ and width $W$; the characteristic aspect ratio is $a=h_{0} / W$ (see Fig. 1a). The cell is either slowly converging or diverging, which

${ }^{1}$ Department of Chemical and Biological Engineering, Princeton University, Princeton, New Jersey 08544, USA, ${ }^{2}$ Department of Mechanical and Aerospace Engineering, Princeton University, Princeton, New Jersey 08544, USA. *e-mail: hastone@princeton.edu. 
a

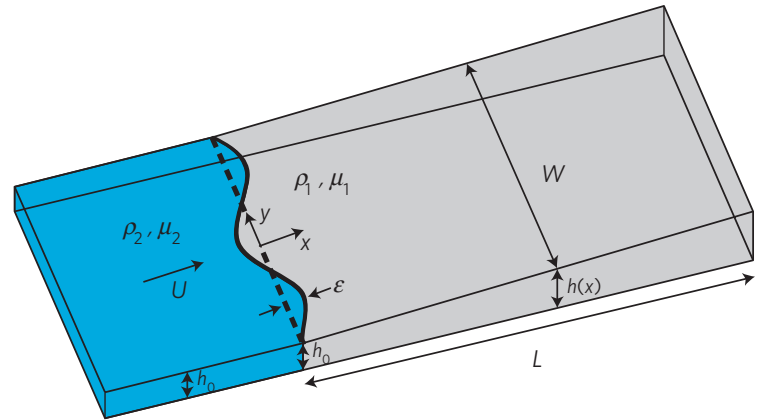

b

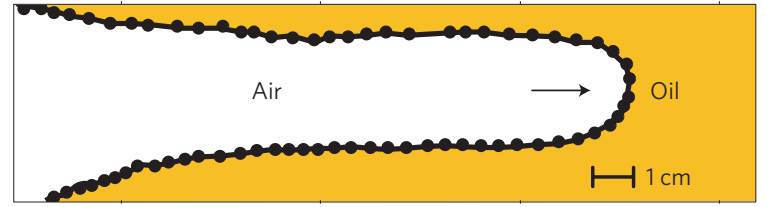

c

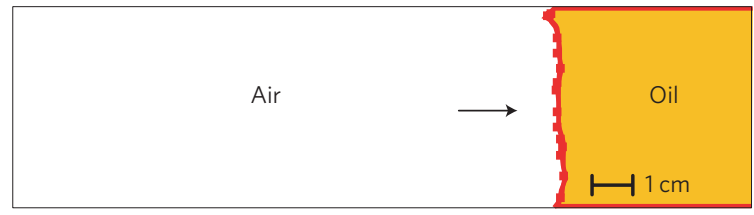

Figure 1 | A variant of classical viscous fingering. a, A schematic of the experimental set-up of a non-uniform Hele-Shaw cell with a constant depth gradient. The depth of the cell $h(x)$, with $h(x) \ll W$ and $h(x) \ll L$, varies linearly in the direction of fluid motion; $d h / d x=\alpha$. $\mathbf{b}$, Top view of a viscous fingering experiment where air displaces mineral oil (viscosity $\mu_{1}=25 \mathrm{cP}$ ) in a horizontal, plexiglass Hele-Shaw cell with a uniform gap of $0.5 \mathrm{~mm}$. The arrows indicate the direction of fluid displacement. The air finger occupies half the width of the cell. c, A stabilized air-oil interface in an experiment conducted in a converging Hele-Shaw cell, $\alpha=-2.7 \times 10^{-3}$.

sets the sign of the gradient $\alpha$. Fluid 1 with density $\rho_{1}$ and viscosity $\mu_{1}$ is displaced by fluid 2 with density $\rho_{2}$ and viscosity $\mu_{2}$. The fluids are immiscible. As the experiments are conducted in a nearly horizontal Hele-Shaw cell, the effect of gravity is neglected. As $h(x) \ll W$ and $h(x) \ll L$, the governing equations of the system are the depth-averaged Darcy law, and, the mass conservation equation modified to account for the depth gradient:

$$
\mathbf{u}_{j}=-\frac{h^{2}}{12 \mu_{j}} \nabla p_{j} \quad \text { and } \quad \nabla \cdot\left(h \mathbf{u}_{j}\right)=0
$$

where $p_{j}$ and $\mathbf{u}_{j}$ are, respectively, the depth-averaged pressure and velocity of phase $j$.

The linear stability of the system in time $t$, described by equation (1), is examined by perturbing a uniform interface (see Fig. 1) advancing at a velocity $U$ with a small-amplitude mode in the transverse direction $y \propto \mathrm{e}^{i(k / W) y+\sigma t}$, where $\sigma$ and $k$, respectively, are the growth rate and the dimensionless wavenumber. The kinematic boundary condition ensures that both fluids move at the same velocity at the interface. In addition, the Young-Laplace equation describes the pressure drop at the interface due to surface tension $\gamma$. In the limit of a small gradient, $|\alpha| \ll 1$, the dispersion relation $\sigma(k)$ is

$$
(1+\lambda) \frac{W}{U} \sigma=\left(1-\lambda+\frac{2 \alpha \cos \theta_{\mathrm{c}}}{\mathrm{Ca}}\right) k-\frac{a^{2}}{\mathrm{Ca}} k^{3}
$$

Here, $\lambda=\mu_{2} / \mu_{1}$ represents the viscosity contrast of the fluids (see Supplementary Information for a more detailed derivation). The

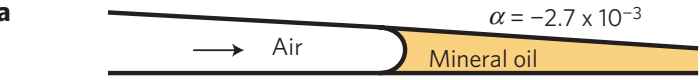

b

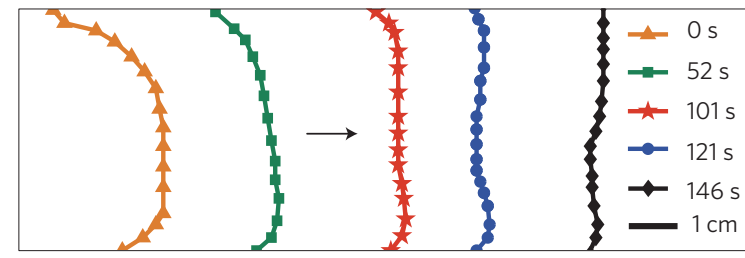

c

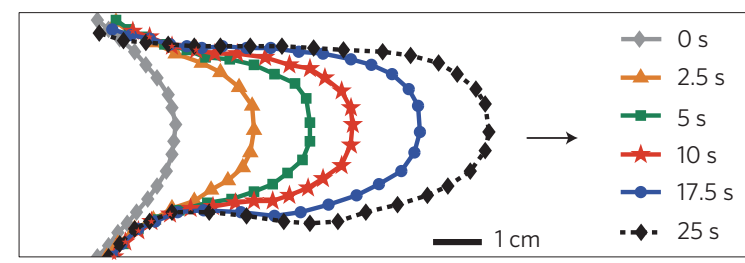

d

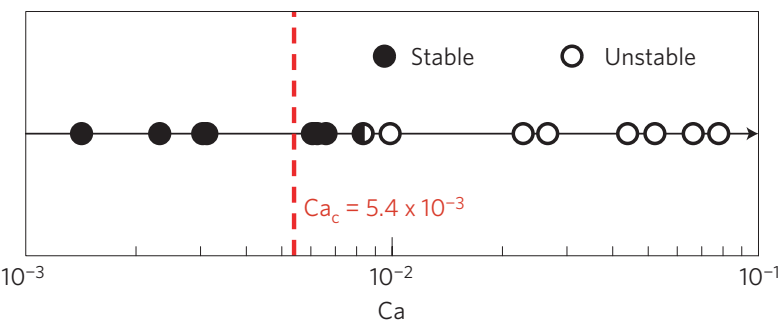

Figure $\mathbf{2}$ | Inhibiting fingering. a, A schematic side view of the experimental set-up where non-wetting air displaces wetting mineral oil ( $\mu_{1}=25 \mathrm{cP}, \gamma=29 \mathrm{mN} \mathrm{m}^{-1}$ ) in a converging Hele-Shaw cell $\left(h_{0}=1.4 \mathrm{~mm}\right.$, $\left.\alpha=-2.7 \times 10^{-3}\right)$. The arrows indicate the direction of fluid displacement. b. Top view of the evolution of an experiment where viscous fingering is inhibited at a low capillary number $\mathrm{Ca}=6 \times 10^{-3}$. c, Top view of the evolution of an experiment where the interface is unstable at a higher capillary number $\mathrm{Ca}=1.6 \times 10^{-2}$. d, A stability diagram showing that the interface transitions from unstable (open circle) to stable (filled circle) at a critical capillary number $\mathrm{Ca}_{c}$, which is found experimentally (half-filled circle) and predicted theoretically (red dashes) using the stability criterion in equation (3). In contrast, for this fluid combination, the interface is always unstable in the absence of the gradient. See Supplementary Information for movies of Fig. $2 b, c$.

contact angle at the interface $\theta_{c}$ is measured between the plate and the curved meniscus; $\theta_{c}=0$ and $\theta_{c}=\pi$ correspond, respectively, to the cases where the displaced fluid is perfectly wetting (Fig. 2a) and perfectly non-wetting (Fig. 3a).

The contribution of the gradient in cell depth to the dispersion relation in equation (2) appears only in the quantity $2 \alpha \cos \theta_{c} / \mathrm{Ca}$. In the absence of the gradient, that is, $\alpha=0$, the dispersion relation in equation (2) reduces to well-known results ${ }^{1,2,5}$. In contrast, the gradient now affects the behaviour of the interface, which is stable, that is, $\sigma<0$ for all $k>0$, if

$$
1-\lambda+\frac{2 \alpha \cos \theta_{c}}{\mathrm{Ca}} \leq 0
$$

By setting the left-hand side of the stability criterion in equation (3) to zero, we can estimate a critical capillary number $\mathrm{Ca}_{\mathrm{c}}=$ $\left(2 \alpha \cos \theta_{c}\right) /(\lambda-1)$, at which the interface undergoes a change in stability. For a given $\lambda, \mathrm{Ca}_{\mathrm{c}}$ is of the order of $\alpha$. Thus, we have established a critical speed, dependent on $\alpha$, for the transition 
a

$\longrightarrow$ Silicon oil $\quad$ Water

b
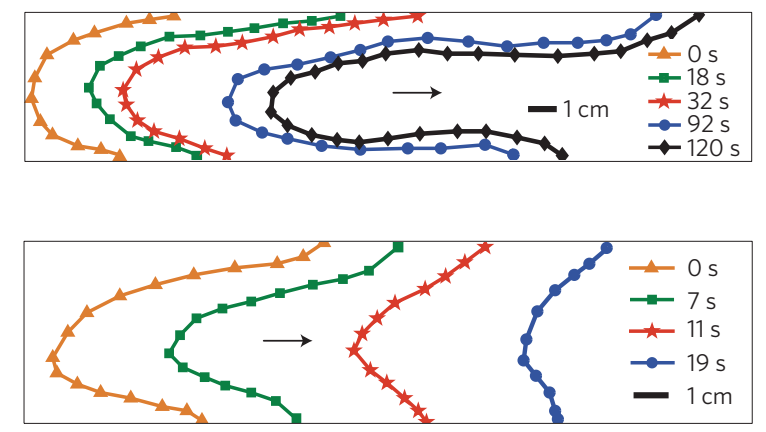

d

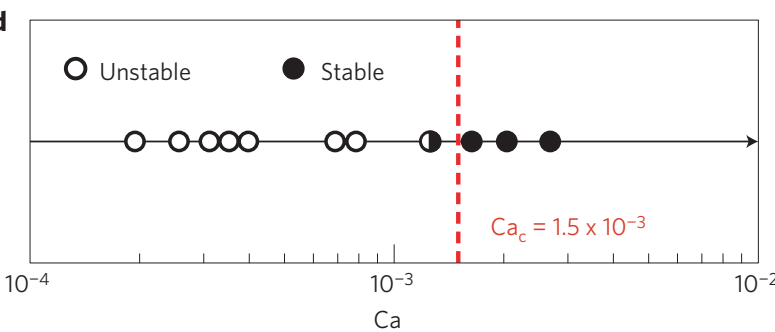

Figure 3 | Triggering fingering. a, A schematic side view of the experimental set-up where wetting silicon oil $\left(\mu_{2}=4.6 \mathrm{cP}\right)$ displaces non-wetting water $\left(\mu_{1}=1 \mathrm{cP}, \gamma=40.5 \mathrm{mN} \mathrm{m}^{-1}\right)$ in a converging Hele-Shaw cell $\left(h_{0}=1.8 \mathrm{~mm}, \alpha=-2.7 \times 10^{-3}\right)$. The arrows indicate the direction of fluid displacement. $\mathbf{b}$, Top view of the evolution of an experiment where fingering is triggered at a low capillary number $\mathrm{Ca}=4 \times 10^{-4}$. c, Top view of the evolution of an experiment conducted at a higher capillary number $\mathrm{Ca}=1.6 \times 10^{-3}$. The system is stable because disturbances decay in time. $\mathbf{d}$, A stability diagram showing that the interface transitions from stable (filled circle) to unstable (open circle) at a critical capillary number $\mathrm{Ca}_{\mathrm{c}}$, which is found experimentally (half-filled circle) and predicted theoretically (red dashes) using the stability criterion in equation (3). In contrast, for this fluid combination, the interface is always stable in the classical, uniform Hele-Shaw cell.

between stable and unstable displacements. Our prediction agrees with the air-oil experiments (Fig. 2) that reveal that the interface is stable below a threshold (Fig. 2d). We note that our analysis assumes a static contact angle and also does not account for the effect of the thin wetting film trailing behind the displaced fluid $^{31}$. We believe that these effects are responsible for the modest discrepancies between the theoretical and experimental thresholds.

By examining equation (3), it is also evident that we can use the effect of the gradient in dictating the stability of the interface in a narrow passage. To confirm this idea, and as we have previously inhibited fingering (Fig. 2), we seek to trigger fingering instabilities in a classically stable system. Figure 3 shows experiments where wetting silicon oil is displacing non-wetting water in a converging Hele-Shaw cell. For this choice of liquids, the interface would be stable in a uniform Hele-Shaw cell because the viscosity ratio of the fluids $\lambda=4.6>1$ (refs 1,2,5). However, in the presence of a negative depth gradient, the interface transitions from stable at high capillary numbers (Fig. 3c) to unstable at low capillary numbers (Fig. 3b). With the invading fluid now being the wetting fluid, the side walls of the Hele-Shaw cell are wetted and this effect acts as an interfacial perturbation, which at low Ca develops into a fingering instability along the cell boundaries (Fig. 3b), that is, where the interfacial perturbations are introduced. Nevertheless, at higher $\mathrm{Ca}$, the interface is stable and a nearly uniform propagating front is observed (Fig. 3c). Again, the stability criterion predicts well the critical capillary number $\mathrm{Ca}_{\mathrm{c}}$ (Fig. 3d) that characterizes the transition from the stable to the unstable regime.

The control of interfacial instabilities is similarly applicable in expanding narrow passages. For instance, our results suggest that when a less viscous fluid displaces a more viscous fluid $(\lambda<1)$ in a cell with a positive gradient, fingering is inhibited provided that the invading fluid is wetting and that the system is operating below a critical flow rate, which corresponds to a critical capillary number.

We have demonstrated that in a narrow passage of slowly varying depth, the stability of the interface between two fluids is governed not only by the viscosity contrast of the fluids, but also by a single critical parameter comprising fluid properties, flow speed and the shape of the flow passage (equation (3)). Therefore, a contracting/expanding geometry offers the opportunity to control the behaviour of interfaces in multiphase systems, which are widely present in nature and technology. For example, we understand the break-up of a droplet flowing past an obstacle ${ }^{15}$. In addition, if fluid displacements occur in passages with flexible walls, the behaviour of the interface is significantly affected owing to the geometrical gradients that result from wall deformations ${ }^{24}$.

More importantly, our results can be used to adjust a system or a design to control interfacial instabilities. For instance, when air or a low-viscosity solvent is used to flush liquids out of a sensor to prepare for reuse, the channel of the sensor may remain contaminated owing to instabilities that cause liquids to be left behind. However, if the channel is tapered and the flow rate is controlled according to equation (3), the sweep efficiency would be greatly enhanced. Finally, knowing the grain-size distribution and other subsurface properties of petroleum reservoirs can benefit secondary and tertiary oil recovery processes. For example, our results suggest that to increase oil recovery in oil-wet reservoirs, injection and production wells can be positioned such that the sweep flow is driven in the direction of decreasing permeability or decreasing grain size (consistent with a model of ref. 32).

\section{Methods}

The fluid-displacement experiments are conducted between two closely spaced, nearly horizontal and smooth plexiglass plates. The plates are $3 / 4$-inch thick to avoid any bending and to ensure a good control over the depth of the Hele-Shaw cell, which is $5.1 \mathrm{~cm}$ in width. The depth gradient is set using level screws distributed along both sides of the cell; the depth of the cell is controlled within $\approx 0.1 \mathrm{~mm}$. From the outlet of the cell, the displaced fluid (fluid 1) is first injected to fill the cell. The invading fluid (fluid 2) is injected from the inlet at constant pressure, which is controlled by adjusting the air supply pressure or by maintaining a constant hydrostatic level at the inlet. To examine interfacial stabilities, all experiments are started with an interface that is as flat as possible. We use backlighting with a paper diffuser so that the Hele-Shaw cell is uniformly illuminated and the interface is clearly identified. The evolution of the interface is captured using a digital video camera. The images are analysed in ImageJ and MATLAB to track the position of the interface and extract its behaviour. The average inlet velocity $U$ is determined by calculating the rate of change of the area occupied by the displaced fluid.

Received 18 April 2012; accepted 17 July 2012; published online 12 August 2012

\section{References}

1. Saffman, P. G. \& Taylor, G. I. The penetration of a fluid into a porous medium or Hele-Shaw cell containing a more viscous liquid. Proc. R. Soc. Lond. A 245, 312-329 (1958).

2. Homsy, G. M. Viscous fingering in porous media. Annu. Rev. Fluid Mech. 19, 271-311 (1987).

3. Wooding, R. A. \& Morel-Seytoux, H. J. Multiphase fluid flow through porous media. Annu. Rev. Fluid Mech. 8, 233-274 (1976).

4. Bensimon, D., Kadanoff, L. P., Liang, S., Shraiman, B. I. \& Tang, C. Viscous flows in two dimensions. Rev. Mod. Phys. 58, 977-999 (1986).

5. Chuoke, R. L., van der Meurs, P. \& van der Poel, C. The instability of slow, immiscible, viscous liquid-liquid displacement in permeable media. Trans. AIME 216, 188-194 (1959). 
6. Lenormand, R., Touboul, E. \& Zarcone, C. Numerical models and experiments on immiscible displacements in porous media. J. Fluid Mech. 189, 165-187 (1988).

7. Huh, D. et al. Acoustically detectable cellular-level lung injury induced by fluid mechanical stresses in microfluidic airway systems. Proc. Natl Acad. Sci. USA 104, 18886-18891 (2007)

8. Grotberg, J. B. \& Jensen, O. E. Biofluid mechanics in flexible tubes. Annu. Rev. Fluid Mech. 36, 121-147 (2004).

9. Taylor, G. I. Cavitation of a viscous fluid in narrow passages. J. Fluid Mech. 16, 595-619 (1963).

10. Ruschak, K. J. Coating flows. Annu. Rev. Fluid Mech. 17, 65-89 (1985).

11. Rabaud, M., Michalland, S. \& Couder, Y. Dynamical regimes of directional viscous fingering: Spatiotemporal chaos and wave propagation. Phys. Rev. Lett. 64, 184-187 (1990).

12. Zhao, H., Casademunt, J., Yeung, C. \& Maher, J. V. Perturbing Hele-Shaw flow with a small gap gradient. Phys. Rev. A 45, 2455-2460 (1992).

13. Olbricht, W. L. \& Leal, L. G. The creeping motion of immiscible drops through a converging/diverging tube. J. Fluid Mech. 134, 329-355 (1983)

14. Halpern, D. \& Jensen, O. E. A semi-infinite bubble advancing into a planar tapered channel. Phys. Fluids 14, 431-442 (2002).

15. Protière, S., Bazant, M. Z., Weitz, D. A. \& Stone, H. A. Droplet breakup in flow past an obstacle: A capillary instability due to permeability variations. Europhys. Lett. 92, 54002 (2010).

16. Langer, J. S. Instabilities and pattern formation in crystal growth. Rev. Mod. Phys. 52, 1-28 (1980).

17. Gallaway, J. W. et al. A lateral microfluidic cell for imaging electrodeposited zinc near the shorting condition. J. Electrochem. Soc. 157, A1279-A1286 (2010)

18. Bhaskar, K. R. et al. Viscous fingering of $\mathrm{HCl}$ through gastric mucin. Nature 360, 458-461 (1992).

19. Wollkind, D. J. \& Segel, L. A. A nonlinear stability analysis of the freezing of a dilute binary alloy. Phil. Trans. R. Soc. Lond. A 268, 351-380 (1970).

20. Ben-Jacob, E. et al. Experimental demonstration of the role of anisotropy in interfacial pattern formation. Phys. Rev. Lett. 55, 1315-1318 (1985).

21. Lake, L. W. Enhanced Oil Recovery (Prentice Hall, 1989).

22. Jha, B., Cueto-Felgueroso, L. \& Juanes, R. Fluid mixing from viscous fingering. Phys. Rev. Lett. 106, 194502 (2011).

23. Dias, E., Parisio, F. \& Miranda, J. Suppression of viscous fluid fingering: A piecewise-constant injection process. Phys. Rev. E 82, 067301 (2010).
24. Pihler-Puzović, D., Illien, P., Heil, M. \& Juel, A. Suppression of complex fingerlike patterns at the interface between air and a viscous fluid by elastic membranes. Phys. Rev. Lett. 108, 074502 (2012).

25. Buckley, S. E. \& Leverett, M. C. Mechanism of fluid displacement in sands. Trans. AIME 146, 107-116 (1942).

26. Hill, S. Channeling in packed columns. Chem. Eng. Sci. 1, 247-253 (1952).

27. Gorell, S. B. \& Homsy, G. M. A theory of the optimal policy of oil recovery by secondary displacement processes. SIAM J. Appl. Math. 43, 79-98 (1983).

28. Orr, F. M. \& Taber, J. J. Use of carbon dioxide in enhanced oil recovery. Science 224, 563-569 (1984).

29. Cinar, Y., Riaz, A. \& Tchelepi, H. A. Experimental study of $\mathrm{CO}_{2}$ injection into saline formations. Soc. Pet. Eng. J. 14, 588-594 (2009).

30. Dias, E. \& Miranda, J. Finger tip behavior in small gap gradient Hele-Shaw flows. Phys. Rev. E 82, 056319 (2010).

31. Park, C-W. \& Homsy, G. M. Two-phase displacement in Hele Shaw cells: Theory. J. Fluid Mech. 139, 291-308 (1984).

32. Yortsos, Y. C., Xu, B. \& Salin, D. Phase diagram of fully developed drainage in porous media. Phys. Rev. Lett. 79, 4581-4584 (1997).

\section{Acknowledgements}

The authors thank I. C. Christov, H. E. Huppert, E. J. Hinch, S. Protière, D. Salin and Z. Zheng for their valuable comments and feedback. We thank BP and the Carbon Mitigation Initiative for partial support. P. A. Tsai thanks NSC Taiwan for support under postdoctoral grant no. 98-2917-564-138. T. T. Al-Housseiny is supported by the National Science Foundation Graduate Research Fellowship under grant no. DGE-0646086.

\section{Author contributions}

T.T.A-H. and H.A.S. conceived the project. T.T.A-H. and P.A.T. performed the experiments and analysed the data. T.T.A-H. and H.A.S. performed the theoretical calculations. All authors participated in writing the manuscript.

\section{Additional information}

Supplementary information is available in the online version of the paper. Reprints and permissions information is available online at www.nature.com/reprints. Correspondence and requests for materials should be addressed to H.A.S

\section{Competing financial interests}

The authors declare no competing financial interests. 